\title{
Mammary Carcinoma Arising in Microglandular Adenosis: A Report of Five Cases
}

\author{
Mimi Kim ${ }^{1} \cdot$ Milim Kim $^{1,2}$ \\ Yul Ri Chung ${ }^{1}$. So Yeon Park Pa $^{1,2}$ \\ 'Department of Pathology, Seoul National \\ University College of Medicine, Seoul; \\ 2Department of Pathology, Seoul National \\ University Bundang Hospital, Seongnam, Korea \\ Received: October 11, 2016 \\ Revised: November 8, 2016 \\ Accepted: November 11, 2016 \\ Corresponding Author \\ So Yeon Park, MD, PhD \\ Department of Pathology, Seoul National University \\ Bundang Hospital, 82 Gumi-ro 173beon-gil, \\ Bundang-gu, Seongnam 13620, Korea \\ Tel: +82-31-787-7712 \\ Fax: +82-31787-4012 \\ E-mail: sypmd@snu.ac.kr
}

\begin{abstract}
Mammary carcinoma arising in microglandular adenosis (MGA) is extremely rare, and MGA is regarded as a non-obligate precursor of triple-negative breast cancer. We report five cases of carcinoma arising in MGA of the breast. All cases showed a spectrum of proliferative lesions ranging from MGA to atypical MGA, ductal carcinoma in situ or invasive carcinoma. Immunohistochemically, all cases were triple-negative and expression of S-100 protein gradually decreased as the lesions progressed from MGA to atypical MGA and carcinoma. Three cases showed acinic cell differentiation with reactivity to $\alpha 1$-antitrypsin, and one case was metaplastic carcinoma. During clinical follow-up, one patient developed local recurrence. Carcinoma arising in MGA is a rare but distinct subset of triple-negative breast cancer with characteristic histologic and immunohistochemical findings.
\end{abstract}

Key Words: Microglandular adenosis; Breast; Carcinoma
Microglandular adenosis (MGA) is a rare benign breast lesion, and it often mimics invasive carcinoma due to the lack of myoepithelium and the infiltrative growth pattern in the stroma. ${ }^{1}$ The unique immunohistochemical characteristic of MGA is the positivity for S-100 protein which is not generally positive in normal breast ductal and glandular cells.

Carcinomas arising in MGA (MGACA) are reported to comprise $27 \%$ of all MGA cases. ${ }^{2}$ They are well known for its various histologic manifestations, showing apparent tumor heterogeneity as in the mixed carcinomas. ${ }^{3-5}$ The other proliferative entity related to MGA, whose atypia is insufficient to be classified as carcinoma, is classified as atypical MGA (AMGA). Whether AMGA is a precursor of the MGACA is still under discussion and the evidences from molecular and genetic analyses for this topic are being accumulated.

This case report presents five cases of MGACA focusing on some of the interesting histologic features and immunohistochemical findings to further clarify the clinicopathologic characteristics of this rare entity.

\section{CASE REPORT}

By searching the electronic database, we found five cases of MGACA (including an in situ lesion). The clinical characteristics of these five are summarized in Table 1 . The median age of the patients at the time of the diagnosis was 47 years (range, 40 to 60 years). All patients underwent breast conserving surgery. Two patients had lymph node metastasis on pathologic examination and four received adjuvant chemo-radiation therapy. During follow-up, one patient (case 1) developed recurrence in the ipsilateral breast and axillary lymph nodes 1 year after the surgery. The rest of the patients had no evidence of disease and are being regularly followed up. This study was approved by the Institutional Review Board of Seoul National University Bundang Hospital (protocol \# B-1609-364-701), and informed consent was waived.

The pathologic findings and the results of the immunohistochemical studies are shown in the Table 2. One patient (case 5) was diagnosed as ductal carcinoma in situ (DCIS) and the rest of the cases were all invasive carcinomas. The lesions in these five cases displayed the histologic spectrum ranging from MGA to 
Table 1. Clinical characteristics of the five cases of MGACA

\begin{tabular}{|c|c|c|c|c|c|}
\hline Characteristic & Case 1 & Case 2 & Case 3 & Case 4 & Case 5 \\
\hline Age (yr) & 40 & 38 & 47 & 46 & 60 \\
\hline Location & Left & Left & Left & Left & Left \\
\hline T stage (size of tumor, cm) & $\mathrm{T} 1 \mathrm{c}(2.0)$ & T2 (2.3) & $\mathrm{T} 1 \mathrm{c}(1.2)$ & T2 (2.5) & Tis (4.5) \\
\hline N stage & $\mathrm{N} 1 \mathrm{a}$ & NO & $\mathrm{N} 1 \mathrm{a}$ & NO & NO \\
\hline Type of surgery & BCS & BCS & BCS & BCS & BCS \\
\hline Adjuvant CRTx & Received & Received & Received & Received & Not received \\
\hline Disease status & Local recurrence & NED & NED & NED & NED \\
\hline
\end{tabular}

MGACA, carcinoma arising in microglandular adenosis; BCS, breast-conserving surgery; CRTx, chemo-radiation therapy; NED, no evidence of disease.

Table 2. Pathologic and immunohistochemical features of the five cases of MGACA

\begin{tabular}{|c|c|c|c|c|c|}
\hline Characteristic & Case 1 & Case 2 & Case 3 & Case 4 & Case 5 \\
\hline Histologic subtype & $\begin{array}{l}\text { Invasive carcinoma, } \\
\text { NST }\end{array}$ & $\begin{array}{l}\text { Invasive carcinoma, } \\
\text { NST }\end{array}$ & $\begin{array}{l}\text { Invasive carcinoma, } \\
\text { NST }\end{array}$ & Metaplastic carcinoma & $\begin{array}{l}\text { Ductal carcinoma } \\
\text { in situ }\end{array}$ \\
\hline Acinic cell differentiation & Absent & Present & Present & Absent & Present \\
\hline Estrogen receptor & Negative & Negative & Negative & Negative & Negative \\
\hline Progesterone receptor & Negative & Negative & Negative & Negative & Negative \\
\hline HER-2 & Negative & Negative & Negative & Negative & Negative \\
\hline S-100 protein & Decreased & Decreased & Decreased & Decreased & Intact \\
\hline Cytokeratin 5/6 & Positive & Positive & Positive & Negative & Positive \\
\hline p53 & Negative & Negative & Negative & Positive & Negative \\
\hline Ki-67 index (\%) & 50 & 20 & 30 & 80 & 5 \\
\hline a1-Antitrypsin & Negative & Positive & Positive & Negative & Positive \\
\hline Chymotrypsin & Negative & Negative & Negative & Negative & Negative \\
\hline
\end{tabular}

MGACA, carcinoma arising in microglandular adenosis; NST, no special type; HER-2, human epidermal growth factor receptor 2.

AMGA and finally to MGACA (Fig. 1). The MGA areas had the typical structure: groups of small round glands infiltrating the stroma of the breast and adipose tissue. Glands were single layered and lined by an intact basement membrane, which can be highlighted by periodic acid-Schiff (PAS) and reticulin stains. However, myoepithelium was absent, as revealed by the absence of immunohistochemical reactivity for calponin. In the lumens of the glands, eosinophilic secretions were common.

In areas diagnosed as AMGA, glands became irregular in shape and cytologically, the nuclear pleomorphism or atypism increased as the amount of eosinophilic secretions decreased. Furthermore, the proliferating atypical cells filled the lumens completely to form carcinoma in situ lesions and finally grew to break the basement membrane to invade the stroma which can be visualized as interrupted and discontinued strands of fibers on PAS and reticulin stains.

Even though this histological spectrum occurred in all four cases of the invasive cancers, the invasive components of some cases had unique morphologic features to be sub-classified separately. Cases 2 and 3 had some characteristic features in the glands, which were similar to those of the pancreas or of the salivary gland. These specific glands tend to make acinar structures with abundant eosinophilic cytoplasmic granules. Case 5, the
DCIS lesion, also showed acinic cell differentiation. The acinic cell differentiation in these three cases was confirmed by the immunohistochemical staining for $\alpha 1$-antitrypsin and chymotrypsin (Table 2, Fig. 2). Case 4 was diagnosed as metaplastic carcinoma, specifically a matrix-producing carcinoma showing an abrupt transition from the typical invasive carcinoma to spindle cell carcinoma with chondromyxoid matrix.

All tumor cells of MGACA in our cases were negative to estrogen receptor, progesterone receptor, and human epidermal growth factor receptor 2, that is, triple-negative. For the immunohistochemical study with S-100 protein, which is the most well-known immunohistochemical marker for MGA lesions, we used a polyclonal antibody which is mainly reactive to S-100b subunit. The reactivity gradually decreased as the lesions progressed from MGA to AMGA and MGACA, showing near negativity in some areas of invasive carcinoma (Fig. 3). On the other hand, Ki-67 index increased form MGA to AMGA and finally to MGACA, as expected. p 53 reactivity was negative in four cases except for case 4 . For cytokeratin $5 / 6$, four cases including one DCIS case demonstrated at least focal positivity (Table 2). 

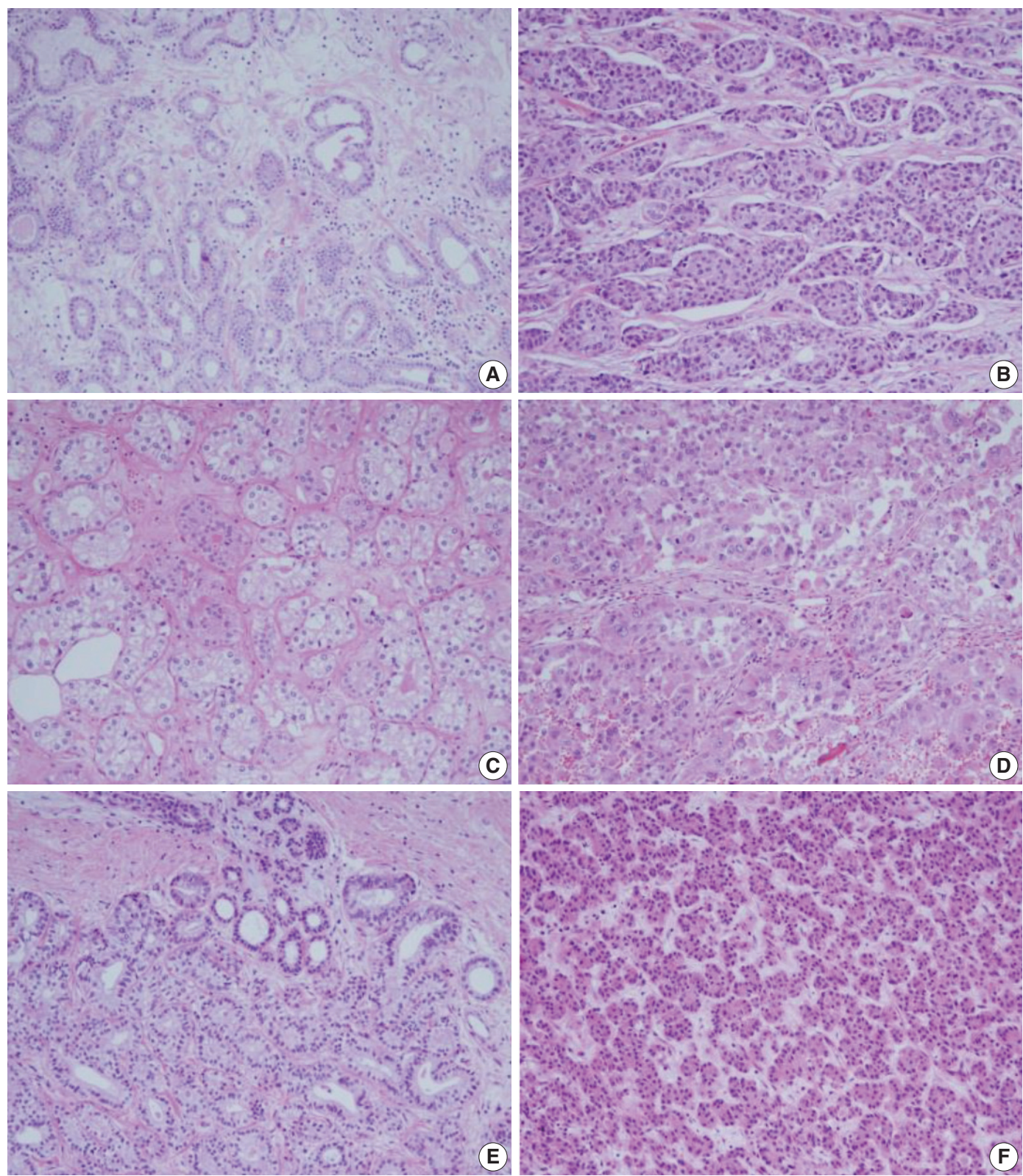

Fig. 1. Representative histologic features of the five cases. (A, C, E, G, I) Microglandular adenosis (MGA) or atypical MGA component. (B, D, $F, H, J)$ Carcinoma component arising in MGA in each case. $(H)$ Histologic features of matrix forming metaplastic carcinoma. (J) Ductal carcinoma in situ (A, B, case 1; C, D, case 2; E, F, case 3; G, H, case 4; I, J, case 5).

(Continued to the next page)

\section{DISCUSSION}

The MGA differs substantially from other adenosis lesions in many aspects including the immunohistochemical properties. The myoepithelial linings, which disappear in MGA, resulted in negative reactivity to the myoepithelial markers such as calponin and $\mathrm{p} 63$. In our case, calponin was all negative in the MGA lesions as well as in the associated carcinoma. The PAS and reticulin staining instead revealed the intact and regular linings of the basement membranes of MGA. These staining patterns therefore could be used to distinguish the invasive portion from the DCIS or other AMGA lesions. The invasive cancers showed the irregular and discontinuous stromal fibers around tumor cell nests.

Through the immunohistochemical studies, we could detect some phenotypic changes from MGA to MGACA. Ki-67 index increased in a stepwise manner as the diseases progressed. Especially in case 4, p53 overexpression gradually increased during transition from AMGA to MGACA. In contrast, the positivity 

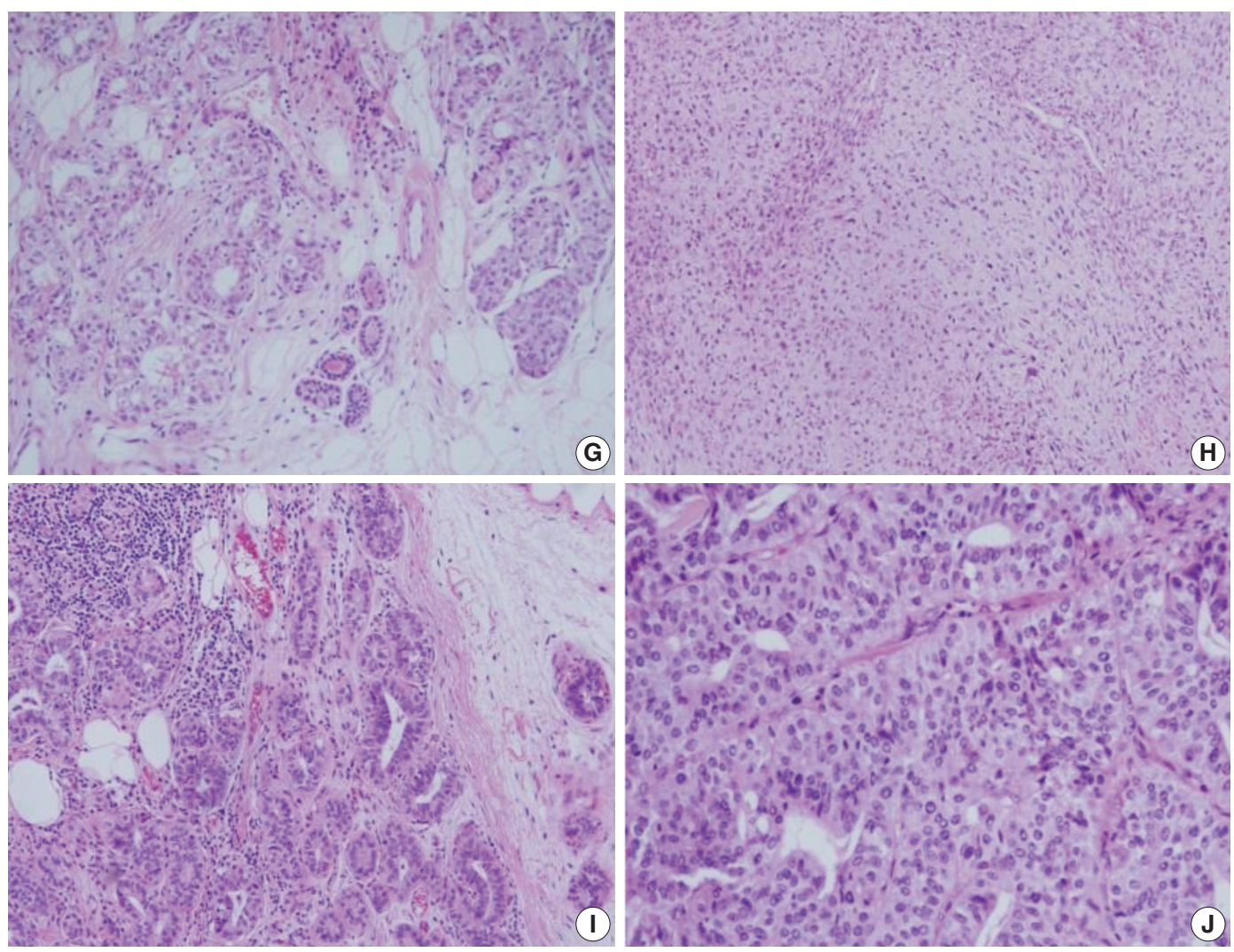

Fig. 1. (Continued from the previous page)

of S-100 protein, the most unique immunohistochemical property of MGA, diminished gradually as the disease progressed to MGACA.

However, some studies reported positive results with S-100 protein in carcinoma portions as strong as in the pure MGA lesions. ${ }^{5,6}$ This discordance may have resulted from the use of different members of S-100 protein, which is known to have at least 21 types currently. In a study of MGACA, Koenig et al. ${ }^{3}$ used two separate antibodies of S-100 protein, S-100a and S-100b for immunohistochemical characterization of MGACA, and reported that S-100b protein showed decreased reactivity, which is similar to our result. They suggested that this finding might be derived from the different localizations of the S-100a and S-100b, which are ductal cytoplasm and myoepithelium, respectively. ${ }^{3}$ However, as MGA shows immunoreactivity to S-100b, it is plausible that epithelial cells of MGA pose myoepithelial cell features and this characteristic may be lost during the progression from MGA to MGACA.

The various histologic and immunohistochemical features of MGACA in our cases have some points of interest. Among the various molecular subtypes of the triple-negative breast cancer, ${ }^{8}$ the metaplastic carcinoma case resembles mainly the mesenchy- mal subtype because it had chondromyxoid and spindle cell areas and was highly proliferative (Ki-67 index, 80\%). Several MGACAs of our cases demonstrated acinic cell differentiation. Interestingly, two invasive cancers with acinic cell differentiation were all positive for $\alpha 1$-antitrypsin and totally negative for chymotrypsin. The DCIS case with acinic cell differentiation showed weak positivity for $\alpha 1$-antitrypsin. These findings are reminiscent of acinic cell carcinoma of the salivary gland rather than that of the pancreas, which is usually positive for chymotrypsin and $\alpha 1$ antichymotrypsin and almost always negative for $\alpha 1$-antitrypsin. It is also consistent with the fact that the traditional acinic cell carcinomas of the breast look similar to that of the salivary gland. Large proportions of the acinic cell carcinomas of the breast reported in articles share many aspects in common with our cases both histologically and immunohistochemically; this suggests that most acinic cell carcinomas originated in breast may arise from MGA lesions.

The molecular characteristics of MGA and MGACA are currently being investigated ${ }^{9-12}$ and one study reported some shared genetic mutations between MGA and MGACA in four out of 12 cases. ${ }^{11}$ The overlapped sequences included recurrent gains on 1q, 2q, and 8q and losses in 14q. They suggested that MGA 

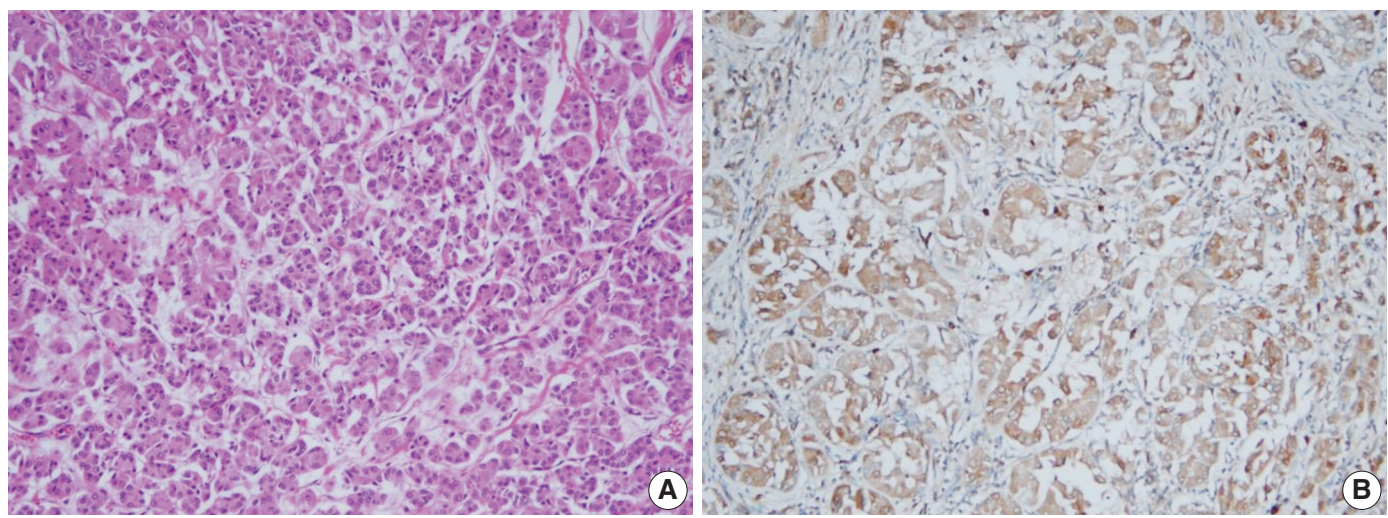

Fig. 2. Acinic cell differentiation in case 3. Tumor cells have eosinophilic granular cytoplasm (A) and show immunoreactivity to $\alpha 1$-antitrypsin (B).
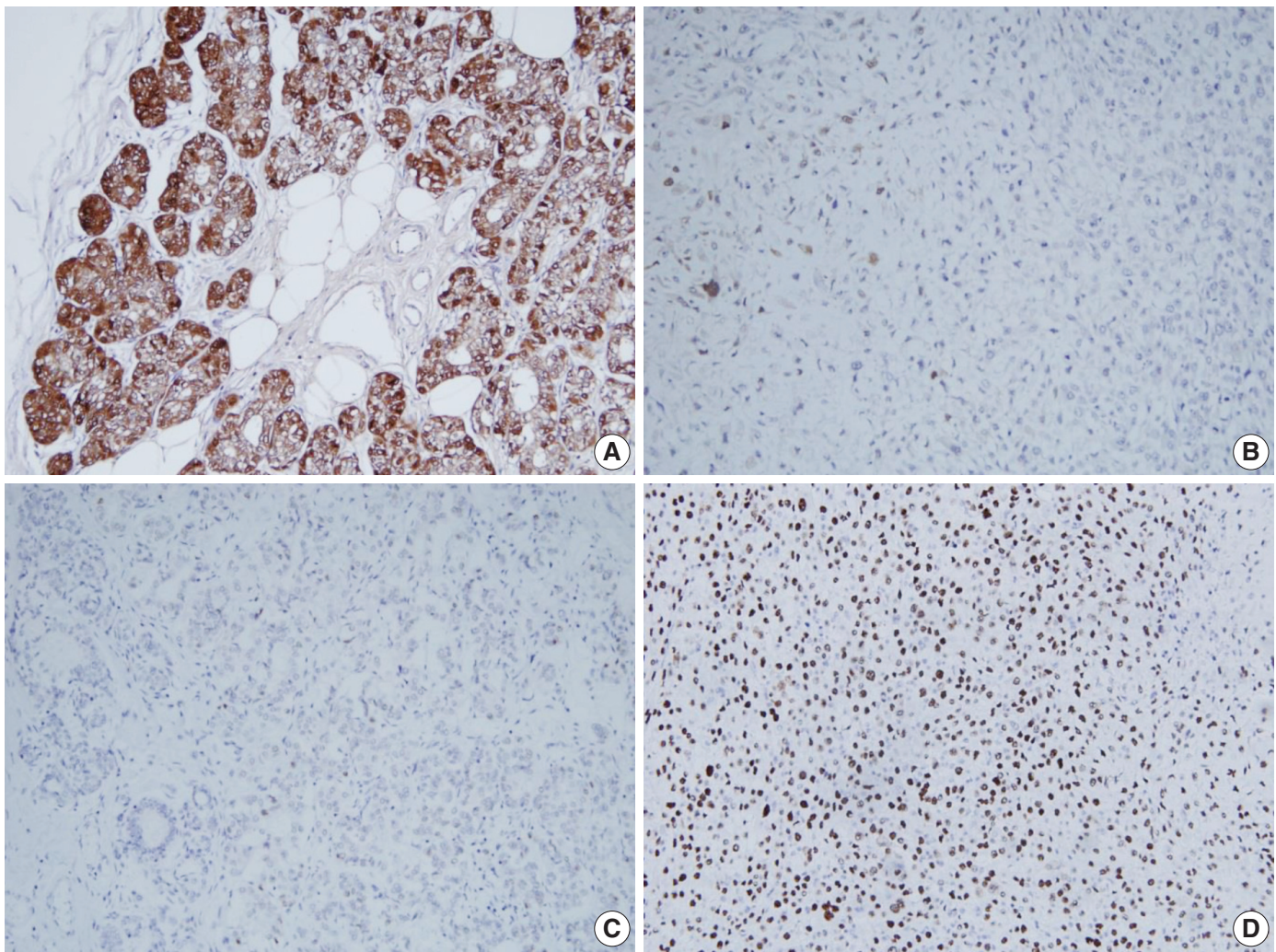

Fig. 3. S-100 protein and p53 expression in case 4. (A, B) Atypical microglandular adenosis (AMGA) shows diffuse strong positivity to S-100 protein (A), while carcinoma arising in microglandular adenosis (MGACA) shows decreased expression to S-100 protein (B). (C, D) p53 staining is evident in MGACA (D), but not in AMGA (C).

might be a non-obligate precursor to a specific subgroup of high grade triple-negative breast carcinomas. ${ }^{11}$

As the histologic spectrum of the MGA associated lesions (from the MGA to AMGA and MGACA) becomes a frequent finding, some researchers suggested a new term, "microglandular adenoma" instead of "microglandular adenosis" for its potential to become a cancer. ${ }^{9}$ According to their opinions, MGA seems to be more similar to the adenoma of the gastrointestinal tract rather than other adenosis lesions of the breast in terms of the possibility to progress to malignancies.

In conclusion, MGACA is one of the rare breast cancers and has many unique features that are worth classifying. Further studies will be needed to find the association between the genetic backgrounds and the characteristics of the lesions. 


\section{Conflicts of Interest}

No potential conflict of interest relevant to this article was reported.

\section{REFERENCES}

1. Rosen PP. Rosen's breast pathology. 2nd ed. Philadelphia: Lippincott Williams \& Wilkins, 2001.

2. Rosenblum MK, Purrazzella R, Rosen PP. Is microglandular adenosis a precancerous disease? A study of carcinoma arising therein. Am J Surg Pathol 1986; 10: 237-45.

3. Koenig C, Dadmanesh F, Bratthauer GL, Tavassoli FA. Carcinoma arising in microglandular adenosis: an immunohistochemical analysis of 20 intraepithelial and invasive neoplasms. Int J Surg Pathol 2000; 8: 303-15.

4. Khalifeh IM, Albarracin C, Diaz LK, et al. Clinical, histopathologic, and immunohistochemical features of microglandular adenosis and transition into in situ and invasive carcinoma. Am J Surg Pathol 2008; 32: 544-52.

5. Shui R, Bi R, Cheng Y, Lu H, Wang J, Yang W. Matrix-producing carcinoma of the breast in the Chinese population: a clinicopathological study of 13 cases. Pathol Int 2011; 61: 415-22.
6. Shui $\mathrm{R}$, Yang $\mathrm{W}$. Invasive breast carcinoma arising in microglandular adenosis: a case report and review of the literature. Breast $\mathrm{J}$ 2009; 15: 653-6.

7. Marenholz I, Heizmann CW, Fritz G. S100 proteins in mouse and man: from evolution to function and pathology (including an update of the nomenclature). Biochem Biophys Res Commun 2004; 322: 1111-22.

8. Lehmann BD, Bauer JA, Chen X, et al. Identification of human triple-negative breast cancer subtypes and preclinical models for selection of targeted therapies. J Clin Invest 2011; 121: 2750-67.

9. Geyer FC, Kushner YB, Lambros MB, et al. Microglandular adenosis or microglandular adenoma? A molecular genetic analysis of a case associated with atypia and invasive carcinoma. Histopathology 2009; 55: 732-43.

10. Shin SJ, Simpson PT, Da Silva L, et al. Molecular evidence for progression of microglandular adenosis (MGA) to invasive carcinoma. Am J Surg Pathol 2009; 33: 496-504.

11. Geyer FC, Lacroix-Triki M, Colombo PE, et al. Molecular evidence in support of the neoplastic and precursor nature of microglandular adenosis. Histopathology 2012; 60: E115-30.

12. Tsang JY, Tse GM. Microglandular adenosis: a prime suspect in triple-negative breast cancer development. J Pathol 2016; 239: 129-32. 